\title{
Spatial Instability of Boundary Layer Along Impedance Wall
}

\author{
Sjoerd W. Rienstra* \\ Eindhoven University of Technology, 5600 MB Eindhoven, The Netherlands. \\ Gregory G. Vilenski ${ }^{\dagger}$ \\ University of Manchester, Manchester, UK.
}

\begin{abstract}
A numerical analysis is made of the hydro-acoustical spatial instability, apparently occurring in a mean flow with thin boundary layer along a locally reacting lined duct wall. This problem is of particular interest because unstable behaviour of liner and mean flow has been observed only very rarely.

It is found that this instability quickly disappears for increasing boundary layer thickness. Specifically, for boundary-layer-thickness based Helmholtz numbers $\omega \delta / c_{0}$ of the order of 0.1 the growth rate vanishes and the instability disappears. This corresponds to very thin boundary layers for practical values of frequencies that occur in aero-engine applications, which is in turn in good agreement with the fact that in industrial practice no instabilities are observed.

For low duct-radius based Helmholtz numbers $(\sim 1)$, the instability exists for rather large values of $\delta$ as an almost neutrally stable wave. This is qualitatively in good agreement with the experimental observations of Ronneberger and Auregan.

It is shown by a Rayleigh-type stability criterion that impedance related hydrodynamic instabilities of temporal type do not occur for mean flows with strictly negative 2nd derivative (the usual situation).
\end{abstract}

\section{Introduction}

S OunD occurs mostly in the form of very small perturbations, and it seems well justified to model it by some form of linearised Euler equations. Viscous boundary layers of high-Reynolds-number flows along walls are, in the aero-engine applications we have in mind, much thinner than a characteristic wave length. This led Ingard [1] and later Myers [2] to derive their impedance wall condition for sound, of a given frequency, at an impedance wall in a mean flow with slip at the wall (i.e. the limit of a vanishing boundary layer, or the boundary layer being collapsed into a vortex sheet). The aero-acoustics community followed them and now the Ingard-Myers boundary condition is the state-of-the-art for this kind of mean flows. However, more refined analyses [3-6] as well as numerical time-domain results [7-9] indicate that the wall vortex sheet along the lined wall may be spatially unstable. This instability is to be interpreted as related to the Kelvin-Helmholtz instability [10] inherent to vortex sheets separating two inviscid fluids of different velocity. In the context of duct acoustical mode analysis it means that one of the modes, found for a given frequency and azimuthal mode number, apparently running upstream and exponentially decaying, is really to be counted among the downstream running modes and exponentially increasing.

For impedances of not very small resistance (as is usual for aero-engine intake and by-pass liners) the spatial growth rate of this instability is very large, but nevertheless (to the authors' knowledge, [11]) it has never been observed. For liner impedances of small resistance on the other hand, there exists experimental evidence $[12,13]$.

So on the one hand the existence of the instability in the model is real (due to the numerical evidence) but on the other hand it has to be an artifact of the model for most of the chosen impedances.

An important question is therefore: what is the major simplification that produces this very undesirable side-effect. An obvious culprit is the linearisation. Any exponentially growing wall-streamline displacement is in reality quickly hampered by the presence of the wall. Although this will certainly limit any instability, it would not exclude its very first onset. Therefore, it is not a fully satisfying explanation, and we have searched for a better one.

*Associate Professor, Department of Mathematics \& Computer Science, Eindhoven University of Technology, P.O. Box 513, 5600 MB Eindhoven, The Netherlands, s.w.rienstra@tue.nl, AIAA Member.

${ }^{\dagger}$ Research Assoc., School of Mathematics, The University of Manchester, Oxford Road, Manchester M13 9PL, UK, gvilensk@ma.man.ac.uk

Copyright (C) 2008 by S.W. Rienstra \& G.G. Vilenski. Published by the American Institute of Aeronautics and Astronautics, Inc. with permission. 
A rather alarming result was presented recently by Brambley and Peake [14], who showed that the combination of the Ingard-Myers condition for vanishing boundary layers and a seemingly regular and physical locally reacting impedance forms an ill-posed problem, which is only interpretable after suitable regularisation. They showed that a regularisation based on an impedance realisation in the form of a flexible wall (which is of course not locally reacting any more), but with preservation of the Ingard-Myers limit, the system is absolutely unstable. In other words, the traditional models do not just contain spatial instabilities of the type that can be found in any shear flows, but instabilities that grow everywhere.

For this reason we will avoid the Ingard-Myers limit by considering only mean flows without slip at the wall. Furthermore, we present in the Appendix a result that (under assumptions given) tells us when the perturbations are at least not temporal unstable (a limited form of absolute unstable).

Returning to our original question, it should be observed that a problem very similar to ours is the instability of a free shear layer, studied by Michalke [15-17]. He found that for zero and (relatively) very small thicknesses of the shear layer, it is spatially unstable, but for Strouhal numbers $\omega \vartheta / \Delta U$ (based on the momentum thickness $\vartheta$ and mean flow difference $\Delta U$ ) typically of order 1 , the instability growth rate vanishes and the shear layer stabilises. So the boundary layer thickness itself may be the limiting factor for the instability to turn up in practice. Inspired by this insight we investigated the spatial stability behaviour for finite boundary layers along the impedance wall.

Using the Pridmore-Brown model for duct modes of given frequency in shear flow along a lined wall, developed earlier $[18,19]$, we were able to analyse the spatial modes in more detail, in particular as a function of complex frequency. When the axial wave number $k_{i}(\omega) \in \mathbb{C}$ can be traced to the other complex half plane as we let $\omega \in \mathbb{C}$ vary far enough into the complex plane, arguments of causality applied to the time-Fourier transformed field tell us that $k_{i}$ really is an instability [20-23].

\section{The problem}

We assume a $\mathrm{e}^{\mathrm{i} \omega t}$-sign convention, while the exponent is dropped throughout.

\section{A. The model}

Consider the equations of motion of an inviscid non-heat-conducting compressible perfect gas flow inside an infinitely long straight circular duct of radius $d$, supplemented by impedance-type boundary conditions with impedance $Z$ (see Figure 1).

$$
\frac{\partial \rho}{\partial t}+\nabla \cdot(\rho \boldsymbol{v})=0 \quad \frac{\partial \boldsymbol{v}}{\partial t}+\boldsymbol{v} \cdot \nabla \boldsymbol{v}=-\nabla p \quad \frac{\mathrm{d} s}{\mathrm{~d} t}=\frac{c_{v}}{p} \frac{\mathrm{d} p}{\mathrm{~d} t}-\frac{c_{p}}{\rho} \frac{\mathrm{d} \rho}{\mathrm{d} t}=\frac{c_{v}}{p}\left(\frac{\mathrm{d} p}{\mathrm{~d} t}+\gamma p \nabla \cdot \boldsymbol{v}\right)=0,
$$

The sound speed $c$ satisfies $c^{2}=\gamma p / \rho$. We linearise these equations and assume harmonic perturbations of the form

$$
(\boldsymbol{v}, p, \rho)=\left(\boldsymbol{v}_{0}, p_{0}, \rho_{0}\right)+\operatorname{Re}\left[(U, V, W, P, \Upsilon) \mathrm{e}^{\mathrm{i} \omega t-\mathrm{i} k x-\mathrm{i} m \theta}\right],
$$

where $\omega$ is the given excitation frequency, $m$ is the given circumferential wavenumber, $k$ is the unknown complex axial wavenumber, and the complex amplitudes $(U, V, W, P, \Upsilon)$ are unknown functions of $r$.

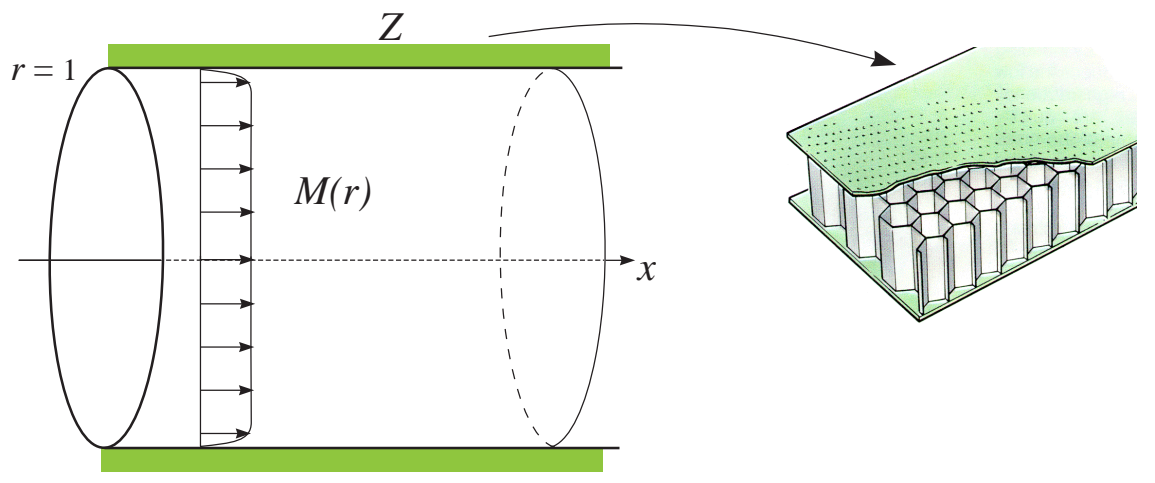

Figure 1. Geometry 
We make the problem dimensionless on duct radius $d$, mean density $\rho_{0}$ and mean sound speed $c_{0}$, and rewrite

$$
\omega:=\frac{\omega d}{c_{0}}, k:=k d, Z:=\frac{Z}{\rho_{0} c_{0}}, \boldsymbol{v}_{0}:=\frac{\boldsymbol{v}_{0}}{c_{0}}, P:=\frac{P}{\rho_{0} c_{0}^{2}}, \quad \boldsymbol{V}:=\frac{\boldsymbol{V}}{c_{0}}, \Upsilon:=\frac{\Upsilon}{\rho_{0}}, t:=\frac{t c_{0}}{d}, \boldsymbol{x}:=\frac{\boldsymbol{x}}{d},
$$

such that $\omega$ is equivalent to the Helmholtz number.

In contrast to the much more general theory of $[18,19]$, the configuration considered here is taken as simple as possible in order obtain canonical and generic results. In particular, the mean flow variables are uniform everywhere except for a $\sim$ tanh-profile of the axial velocity (see Figure 1)

$$
\begin{gathered}
\rho_{0}=1, \quad p_{0}=1 / \gamma, \quad c_{0}=1, \quad w_{0}=0, \quad v_{0}=0, \\
u_{0}(r)=M(r)=M_{0}\left[\tanh \left(\frac{1-r}{\delta}\right)+\left(1-\tanh \left(\delta^{-1}\right)\right)\left(\frac{1+\tanh \left(\delta^{-1}\right)}{\delta} r+(1+r)\right)(1-r)\right] .
\end{gathered}
$$

$M(r)$ is practically constant in $r$ except for a boundary layer of typical thickness $\mathcal{O}(\delta)$; see Figure 2 . For all practical

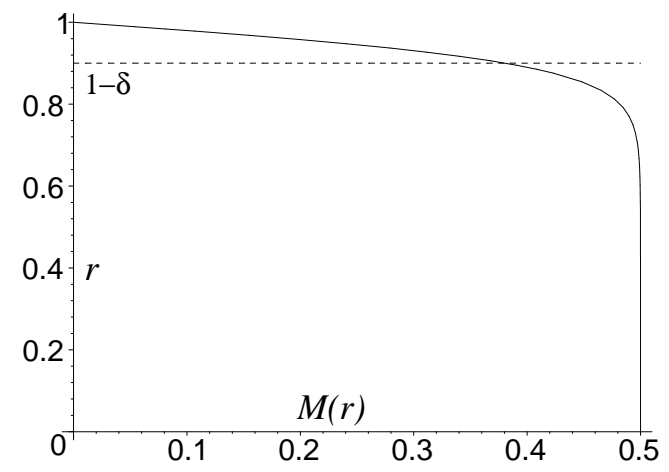

Figure 2. Mean flow profile for $M_{0}=0.1$ and $\delta=0.1$

purposes, $\delta$ is characterised by $M(1-\delta) \simeq 0.76 M_{0}, M(1-3 \delta) \simeq 0.995 M_{0}$, and a momentum thickness given by $\vartheta \simeq(1-\ln 2) \delta \simeq 0.307 \delta$.

The resulting system of equations (note slight notation differences with $[18,19]$ )

$$
\begin{gathered}
\mathrm{i} \lambda \Upsilon+\frac{1}{r}(r V)^{\prime}-\mathrm{i} \frac{m}{r} W-\mathrm{i} k U=0, \quad \mathrm{i} \lambda V=-P^{\prime}, \quad \mathrm{i} \lambda W=\mathrm{i} \frac{m}{r} P, \\
\mathrm{i} \lambda U+u_{0}^{\prime} V=\mathrm{i} k P, \quad \mathrm{i} \lambda P+\frac{1}{r}(r V)^{\prime}-\mathrm{i} \frac{m}{r} W-\mathrm{i} k U=0
\end{gathered}
$$

is reduced to a single equation for $P$, known as the Pridmore-Brown equation [24] for cylindrical coordinates,

$$
P^{\prime \prime}+\left(\frac{1}{r}-\frac{2 \lambda^{\prime}}{\lambda}\right) P^{\prime}+\left(\lambda^{2}-k^{2}-\frac{m^{2}}{r^{2}}\right) P=0
$$

where $\lambda=\omega-k M$ and primes denote differentiation with respect to $r$. The impedance boundary condition is given by

$$
\mathrm{i} \omega Z P^{\prime}=\lambda^{2} P \quad \text { on } r=1 .
$$

This follows Ingard [1] and Myers [2] if $M(1) \neq 0$ and reduces to the regular impedance condition $P=Z V$ if $M(1)=0$. In order to avoid the Ingard-Myers limit here, we will strictly use a mean flow vanishing at the wall. For an impedance to be physical it has to be a function of $\omega$ satisfying certain conditions; see [25]. The form chosen here is simply a mass-spring-damper model

$$
Z(\omega)=R+\mathrm{i} a \omega-\frac{\mathrm{i} b}{\omega}
$$

Note that $a$ and $b$ are made dimensionless on $\rho_{0}, c_{0}$ and duct radius $d$ : when in dimensional form $Z^{\prime}=R^{\prime}+\mathrm{i} a^{\prime} \omega^{\prime}-$ $\mathrm{i} b^{\prime} / \omega^{\prime}$, then $Z^{\prime}=\rho_{0} c_{0} Z, R^{\prime}=\rho_{0} c_{0} R, \omega^{\prime}=\omega c_{0} / d, a^{\prime}=\rho_{0} a d$ and $b^{\prime}=\rho_{0} c_{0}^{2} b / d$. So varying $\omega$ with a fixed impedance model is possible if we vary the physical frequency and leave the duct radius intact. 


\section{B. Numerical method}

The foregoing eigenvalue problem in $k$ was solved numerically by a combination of an implicit finite-difference (backward Euler) scheme for the differential equation and a modified Newton's method to iterate to eigenvalue $k$ as described in $[18,19]$. To the authors' knowledge, this is the only numerical method currently available in the literature which, apart from the acoustic spectrum, allows to accurately and reliably resolve the continuous (i.e., hydrodynamic) part of the spectrum, where the Pridmore-Brown equation is singular. The method is based on the direct mathematical analysis of the structure of the solution of the Pridmore-Brown equation near the singularity. It respects the requirements on the permissible smoothness of the solution at the singular point and utilises the correct condition for continuation of the solution beyond the singularity. As a result, the method does not suffer from the loss of accuracy or numerical stability typical of continuous spectrum computations. In the context of the stability analysis this feature of the method is of crucial importance. It ensures rigorous control over the behaviour of the instability mode and its hydrodynamic counterparts when the trajectory of instability mode crosses the continuous spectrum (see the examples discussed below).

The number of grid points was always large enough to have at least 5 points on the interval $[1-\delta, 1]$ with a minimum overall number of points being 1000. For example, for $\delta=10^{-3}$ (the smallest non-zero value used), 5000 grid points were used. The stop criterion in the Newton search was when the relative change was typically less than $10^{-6}$.

The starting values of eigenvalue $k$ were chosen from a fine-meshed partition ("checkerboard") of the area of interest in the complex $k$-plane.

This procedure has been used to trace the eigenvalues as a function of the complex frequency $\omega=|\omega| \mathrm{e}^{-\mathrm{i} \varphi}$, where $\varphi$ varied in 100 steps from 0 to $\pi / 2$. Most of the found axial wave numbers were just acoustic duct modes and varied only a little bit with $\omega$. If a $k(\omega)$ was found to cross the real axis, the corresponding mode was considered to be a spatial instability.

This is motivated by the observation that if a perturbation quantity is generally given as a time-Fourier integral

$$
\phi(x, t)=\int_{-\infty}^{\infty} \hat{\phi}(x ; \omega) \mathrm{e}^{\mathrm{i} \omega t} \mathrm{~d} \omega
$$

the Fourier transform $\hat{\phi}(\boldsymbol{x} ; \omega)$ has to be analytic in the lower complex $\omega$-half plane $\mathbb{C}_{\omega}^{-}$, for $\phi(\boldsymbol{x}, t)$ to be causal, i.e. vanishing in $t$ for before some time $t_{0}$. This gets entangled with behaviour in the $k$-plane if $\hat{\phi}$ itself is given as an $x$-Fourier integral

$$
\hat{\phi}(x, \omega)=\int_{-\infty}^{\infty} \tilde{\phi}(k, \omega) \mathrm{e}^{-\mathrm{i} k x} \mathrm{~d} k .
$$

If $\tilde{\phi}$ has a pole $k=\kappa(\omega)$ in the upper complex $k$-plane $\mathbb{C}_{k}^{+}$, it is most likely a left-running mode as it contributes to $\hat{\phi}$ for $x<0$, when the $k$-contour can be closed via the upper half plane. The opposite is true for poles in the lower half $k$-plane $\mathbb{C}_{k}^{-}$, contributing as right-running modes. From our results below, this is certainly the case for large enough $|\omega|$, so we have no problems at the ends of the integration contours.

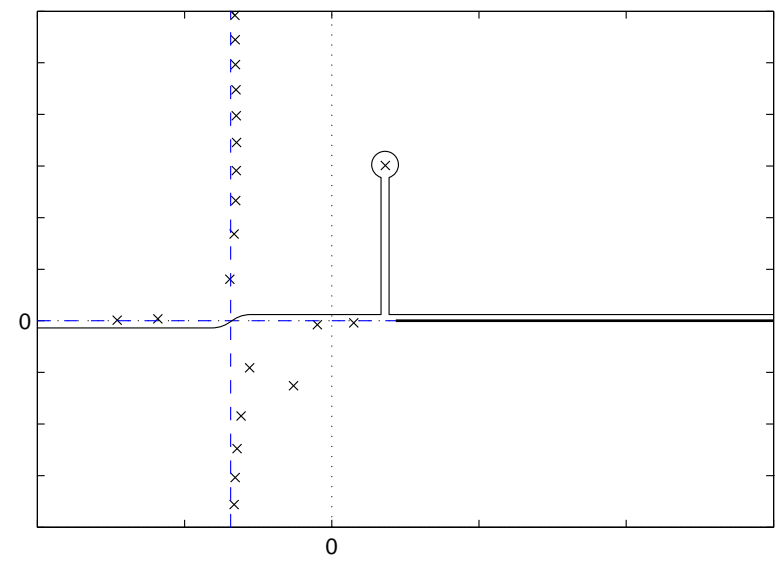

Figure 3. Illustration of deformed $k$-contour 
For smaller $\omega$, however, it may happen that a pole $\kappa_{i}(\omega) \in \mathbb{C}_{k}^{+}$varies with $\omega$ such that for some $\omega \in \mathbb{C}_{\omega}^{-}$it crosses the real $k$-axis (the $k$-contour of integration). This would create a non-analytic and therefore non-causal $\hat{\phi}$. In such cases, the $k$-contour should have been deformed up-around $k=\kappa_{i}(\omega)$ in the first place, and the corresponding mode was apparently a right-running mode, and thus a spatial instability.

Of course, all this relies on various assumptions that has to be sorted out in detail, for example: (i) $\tilde{\phi}$ has no other singularities than poles in the upper and lower complex $k$-halfplanes, while the contribution of the continuous spectrum along the real axis (due to $\lambda$ being zero at $k=\omega / M(r)$ ) is of less importance, and (ii) $|\hat{\phi}(\boldsymbol{x} ; \omega)| \rightarrow 0$ when $|\omega| \rightarrow \infty$ in the lower half plane. See for example [26].

For the moment, whether these assumptions hold cannot be fully substantiated in the present relatively complicated configuration. Our conclusions are therefore to be considered as tentative until further analysis has come available.

\section{Results}

For a number of dimensionless frequencies or Helmholtz numbers $\omega(1-10)$, mean flow Mach numbers $M_{0}(0.1-0.7)$, azimuthal orders $m(0-10)$ and impedances $Z$ of varying resistance, we varied the boundary layer thickness $\delta$ in steps of 0.001 between 0.000 and 0.010 , in some cases 0.050 . When $\delta=0.0$, the result was based on the Ingard-Myers condition. In the other cases the impedance was the same as for no flow.

Figures 4-11 gives examples of the behaviour of the modes as a function of complex $\omega$. First for relatively large $\omega=10$, then down to $\omega=1$. In all figures with $\delta \neq 0$ the continuous spectrum is visible (albeit approximated due to the numerical discretisation), corresponding to (not mode-like) downstream running perturbations.

Figure 10 shows that for small $\omega$ the instability is practically neutral (compared to the other modes is $\operatorname{Im}(k) \simeq 0$ for all $\delta$ ). This agrees with the experimental observations of Ronneberger [12] and Auregan [13].

Figure 12 summarises the behaviour of $\operatorname{Im}(k)$ as a function of $\delta$. Estimates (from the plots) of the found critical $\delta_{c}$ (the $\delta$ where $\operatorname{Im}(k)=0$ ) is tabulated in table 1 .

An other conclusion is the fact that the axial wave number of the instability (if present) is very sensitive for variations in $\delta$, in particular when $\delta$ is small, confirming the earlier conclusions by Tester [3] based on a similar but more restricted exploration of the present problem.

For $\omega=10$ the flow is unstable for $0 \leq \delta \leq \delta_{c} \sim 0.008$, only a little bit depending on the other parameters. For decreasing $\omega$ the critical value $\delta_{c}$ increased, more or less inversely proportionally to $\omega$, to a value of 0.050 for $\omega=1$. Together we seem to have a crude estimate of $\delta_{c}$ given by $\delta_{c} \omega \sim 0.1$, which corresponds to very thin boundary layers for practical values of $\omega=20$ and higher.

All this is qualitatively in good agreement with the fact that in industrial practice no instabilities are observed [11].

\begin{tabular}{rrrlll}
\hline$\omega$ & $m$ & $M$ & \multicolumn{1}{c}{$Z$} & \multicolumn{1}{c}{$\delta_{c}$} & $\omega \delta_{c}$ \\
\hline 10 & 0 & 0.3 & $1.0-0.10 \mathrm{i}$ & 0.012 & 0.12 \\
10 & 0 & 0.5 & $0.1+1.39 \mathrm{i}$ & 0.008 & 0.08 \\
10 & 0 & 0.5 & $1.0+1.39 \mathrm{i}$ & 0.011 & 0.11 \\
10 & 0 & 0.5 & $3.0+1.39 \mathrm{i}$ & 0.01 & 0.1 \\
10 & 5 & 0.4 & $1.0+1.39 \mathrm{i}$ & 0.008 & 0.08 \\
10 & 5 & 0.7 & $1.0+1.39 \mathrm{i}$ & 0.012 & 0.12 \\
10 & 10 & 0.1 & $1.0+1.39 \mathrm{i}$ & 0.009 & 0.09 \\
5 & 0 & 0.1 & $0.1-0.20 \mathrm{i}$ & 0.012 & 0.06 \\
5 & 0 & 0.3 & $2.0-0.20 \mathrm{i}$ & 0.025 & 0.125 \\
5 & 0 & 0.5 & $0.1-0.20 \mathrm{i}$ & 0.045 & 0.225 \\
5 & 0 & 0.5 & $2.0-0.20 \mathrm{i}$ & 0.022 & 0.11 \\
5 & 0 & 0.7 & $2.0-0.20 \mathrm{i}$ & 0.035 & 0.175 \\
5 & 0 & 0.7 & $0.1-0.20 \mathrm{i}$ & 0.05 & 0.25 \\
1 & 0 & 0.1 & $0.1-1.00 \mathrm{i}$ & 0.02 & 0.02 \\
1 & 0 & 0.1 & $1.0-1.00 \mathrm{i}$ & 0.006 & 0.006 \\
1 & 0 & 0.3 & $0.1-1.00 \mathrm{i}$ & 0.03 & 0.03 \\
1 & 0 & 0.3 & $0.5-1.00 \mathrm{i}$ & 0.045 & 0.045 \\
1 & 0 & 0.5 & $0.1-1.00 \mathrm{i}$ & 0.04 & 0.04 \\
1 & 0 & 0.5 & $1.0-1.00 \mathrm{i}$ & 0.06 & 0.06 \\
\hline
\end{tabular}

Table 1. Table of critical $\delta_{c}$, i.e. the $\delta$ with $\operatorname{Im}(k)=0$ (estimated from the plots). 


\section{Appendix}

\section{A Rayleigh-type temporal stability criterion for hydrodynamic instabilities}

In order to make sure that the kind of instabilities we are considering is not temporal, a stability analysis $a$ la Rayleigh's Inflection Point Theorem [10] can be made. If we may assume a hydrodynamic (weakly compressible) approximation, it follows indeed that no temporal instability is possible in 2D parallel mean flow along an impedance wall.

For thin boundary layers and high enough Helmholtz numbers in the duct we consider a 2D parallel flow model, which is in dimensional ${ }^{\text {a }}$ form given by equations for conservation of mass, momentum and entropy and are the same as equations (1). We linearise and assume $t$ and $x$-harmonic modes

$$
u:=u_{0}(y)+U \mathrm{e}^{\mathrm{i} \omega t-\mathrm{i} k x}, \quad v:=V \mathrm{e}^{\mathrm{i} \omega t-\mathrm{i} k x}, \quad p:=p_{0}+P \mathrm{e}^{\mathrm{i} \omega t-\mathrm{i} k x}, \quad \rho:=\rho_{0}+R \mathrm{e}^{\mathrm{i} \omega t-\mathrm{i} k x} .
$$

Introduce

$$
\lambda=\omega-k u_{0}, \quad \lambda^{\prime}=-k u_{0}^{\prime}
$$

to obtain

$$
\begin{array}{r}
\mathrm{i} \lambda R+\rho_{0}\left(-\mathrm{i} k U+V^{\prime}\right)=0 \\
\mathrm{i} \rho_{0} \lambda U+\rho_{0} u_{0}^{\prime} V-\mathrm{i} k P=0 \\
\mathrm{i} \rho_{0} \lambda V+P^{\prime}=0 \\
\mathrm{i} \lambda P+\gamma p_{0}\left(-\mathrm{i} k U+V^{\prime}\right)=0 .
\end{array}
$$

The impedance boundary condition at $y=0$ (with $u_{0}(0)=0$ and so $\lambda(0)=\omega$ ) is now

$$
P=-\rho_{0} c_{0} Z(\omega) V
$$

We may rephrase the problem to one in only $P, R$ or $V$. It appears to be most fruitful to work in $V$, such that we obtain

$$
\begin{gathered}
V^{\prime \prime}+\left(\frac{k u_{0}^{\prime \prime}}{\lambda}-k^{2}\right) V+\frac{1}{c_{0}^{2}}\left(\lambda^{2} V-\frac{2 k u_{0}^{\prime}}{k^{2}-\frac{\lambda^{2}}{c_{0}^{2}}}\left(\lambda V^{\prime}+k u_{0}^{\prime} V\right)\right)=0 \\
{\left[k u_{0}^{\prime}+\left(k^{2}-\frac{\omega^{2}}{c_{0}^{2}}\right) \mathrm{i} c_{0} Z\right] V+\omega V^{\prime}=0 \quad \text { at } y=0 .}
\end{gathered}
$$

We bring the equation into the more usual form by introducing

$$
\omega=k \alpha, \quad \text { so } \quad \lambda=\omega-k u_{0}=-k\left(u_{0}-\alpha\right) .
$$

We obtain

$$
V^{\prime \prime}-\left(\frac{u_{0}^{\prime \prime}}{u_{0}-\alpha}+k^{2}\right) V+\frac{1}{c_{0}^{2}}\left(k^{2}\left(u_{0}-\alpha\right)^{2} V+\frac{2 u_{0}^{\prime}}{1-\frac{\left(u_{0}-\alpha\right)^{2}}{c_{0}^{2}}}\left(\left(u_{0}-\alpha\right) V^{\prime}-u_{0}^{\prime} V\right)\right)=0 .
$$

Multiply by $\bar{V}$, the complex conjugate of $V$, and integrate for $y=0$ to $\infty$ while utilising the boundary condition

$$
\begin{array}{r}
\int_{0}^{\infty}\left[\left|V^{\prime}\right|^{2}+\left(\frac{u_{0}^{\prime \prime}}{u_{0}-\alpha}+k^{2}\right)|V|^{2}-\frac{1}{c_{0}^{2}}\left(k^{2}\left(u_{0}-\alpha\right)^{2}|V|^{2}+\frac{2 u_{0}^{\prime}}{1-\frac{\left(u_{0}-\alpha\right)^{2}}{c_{0}^{2}}}\left(\left(u_{0}-\alpha\right) \bar{V} V^{\prime}-u_{0}^{\prime}|V|^{2}\right)\right)\right] \mathrm{d} y= \\
\frac{1}{\alpha}\left(u_{0}^{\prime}+\mathrm{i} c_{0} k Z\left(1-\frac{\alpha^{2}}{c_{0}^{2}}\right)\right)|V(0)|^{2}
\end{array}
$$

\footnotetext{
${ }^{a}$ Note: in order to ease comparison with literature on the Rayleigh stability theorem, and possible limits that may be taken, we have written this appendix entirely in dimensional form, in contrast to the rest of the paper.
} 
We consider only hydrodynamic-type instabilities, albeit connected to the impedance, and approximate for small $u_{0} / c_{0}$

$$
\int_{0}^{\infty}\left[\left|V^{\prime}\right|^{2}+\left(\frac{u_{0}^{\prime \prime}}{u_{0}-\alpha}+k^{2}\right)|V|^{2}\right] \mathrm{d} y \simeq \frac{1}{\alpha}\left(u_{0}^{\prime}+\mathrm{i} c_{0} k Z\right)|V(0)|^{2}
$$

We now limit our type of solutions to temporal instabilities, i.e. we consider $k$ real positive and $\alpha=\beta-\mathrm{i} \mu$ (for a temporal instability is $\mu>0$ ). Consider the physically possible [25] impedance

$$
Z(\omega)=R+\mathrm{i} a \omega-\mathrm{i} \frac{b}{\omega}
$$

(with real positive defining parameters), and take the imaginary parts of left and righthand sides. We obtain the following necessary condition for a temporally unstable solution to exist

$$
\mu \int_{0}^{\infty} \frac{u_{0}^{\prime \prime}}{\left|u_{0}-\alpha\right|^{2}}|V|^{2} \mathrm{~d} y=\frac{1}{|\alpha|^{2}}\left(\mu u_{0}^{\prime}+c_{0} \beta\left(k R+\frac{2 b \mu}{|\alpha|^{2}}\right)\right)|V(0)|^{2}
$$

Noting that for a typical mean flow like $u_{0} \sim \tanh (y / \delta)$ we have $u_{0}^{\prime} \sim 1-\tanh (y / \delta)^{2}>0$ and $u_{0}^{\prime \prime} \sim-\tanh (y / \delta)(1-$ $\left.\tanh (y / \delta)^{2}\right)<0$, we see that the strictly negative left-hand side can never match the strictly positive right-hand side. So no solution of the present type is possible.

\section{Acknowledgements}

The original version of the computer program was developed under the "Messiaen" European collaborative project (EU Technical Officer Dietrich Knörzer and Coordinator Jean-Louis Migeot, Free Field Technologies).

\section{References}

${ }^{1}$ K.U. Ingard, Influence of Fluid Motion Past a Plane Boundary on Sound Reflection, Absorption, and Transmission, Journal of the Acoustical Society of America 31(7), 1035-1036, 1959

${ }^{2}$ M.K. Myers, On the acoustic boundary condition in the presence of flow, Journal of Sound and Vibration, 71 (3), p.429-434, 1980

${ }^{3}$ B.J. Tester, The Propagation and Attenuation of Sound in Ducts Containing Uniform or "Plug" Flow. Journal of Sound and Vibration 28(2), $151-203,1973$

${ }^{4}$ S.W. Rienstra, A Classification of Duct Modes Based on Surface Waves, Wave Motion, 37 (2), p.119-135, 2003.

${ }^{5}$ S.W. Rienstra and B.T. Tester, An Analytic Green's Function for a Lined Circular Duct Containing Uniform Mean Flow, to appear in Journal of Sound and Vibration, 2008

${ }^{6}$ S.W. Rienstra, Acoustic Scattering at a Hard-Soft Lining Transition in a Flow Duct, Journal of Engineering Mathematics, volume 59(4), 2007.

${ }^{7}$ N. Chevaugeon, J.-F. Remacle and X. Gallez, Discontinuous Galerkin Implementation of the Extended Helmholtz Resonator Impedance Model in Time Domain, 12th AIAA/CEAS Aeroacoustics Conference, Cambridge, MA, 8-10 May 2006

${ }^{8}$ C. Richter, F. Thiele, X. Li, M. Zhuang, Comparison of Time-Domain Impedance Boundary Conditions by Lined Duct Flows, AIAA Paper 2006-2527, 12th AIAA/CEAS Aeroacoustics Conference 2006

${ }^{9}$ C. Richter, F. Thiele, The Stability of Time Explicit Impedance Models, AIAA Paper 2007-3538, 13th AIAA/CEAS Aeroacoustics Conference 2007

${ }^{10}$ P.G. Drazin and W.H. Reid, Hydrodynamic Stability, Cambridge University Press, 2nd edition, 2004

${ }^{11}$ Michael G. Jones (NASA Langley), personal communication, 2007.

${ }^{12} \mathrm{M}$. Brandes and D. Ronneberger, Sound amplification in flow ducts lined with a periodic sequence of resonators, AIAA paper 95-126, 1st AIAA/CEAS Aeroacoustics Conference, Munich, Germany, June 12-15, 1995

${ }^{13}$ Y. Aurégan, M. Leroux, V. Pagneux, Abnormal behavior of an acoustical liner with flow, Forum Acusticum 2005, Budapest.

${ }^{14}$ E.J. Brambley and N. Peake, Surface-Waves, Stability, and Scattering for a Lined Duct with Flow, AIAA 2006-2688, 12th AIAA/CEAS Aeroacoustics Conference 8-10 May 2006, Cambridge, MA

${ }_{15}^{15}$ A. Michalke, On Spatially Growing Disturbances in an Inviscid Shear Layer, Journal of Fluid Mechanics, vol. 23(3), pp. 521-544, 1965

${ }_{16}$ A. Michalke, Survey On Jet Instability Theory, Prog. Aerospace Sci., vol. 21, pp. 159-199, 1984

${ }^{17}$ J. Manera, B. Schiltz, R. Leneveu, S. Caro, J. Jacqmot, S.W. Rienstra, Kelvin-Helmholtz Instabilities Occuring at a Nacelle Exhaust, AIAA paper AIAA-2008-2883, 14th AIAA/CEAS Aeroacoustics Conference, Vancouver, Canada, 5-7 May 2008

${ }^{18}$ G.G. Vilenski and S.W. Rienstra, On Hydrodynamic and Acoustic Modes in a Ducted Shear Flow with Wall Lining, Journal of Fluid Mechanics, 583, 45-70, 2007.

${ }^{19}$ G.G. Vilenski and S.W. Rienstra, Numerical Study of Acoustic Modes in Ducted Shear Flows, Journal of Sound and Vibration, 307(3-5), p.610-626, 2007.

${ }^{20}$ P.C. Clemmow and J.P. Dougherty, Electrodynamics of Particles and Plasmas, Addison Wesley, 1969

${ }^{21}$ R.J. Briggs, Electron-Stream Interaction with Plasmas, Monograph no. 29, MIT Press, Cambridge Massachusetts, 1964

${ }^{22}$ A. Bers, Space-Time Evolution of Plasma Instabilities - Absolute and Convective, Handbook of Plasma Physics: Volume 1 Basic Plasma Physics, edited by A.A. Galeev and R.N. Sudan, North Holland Publishing Company, Chapter 3.2, 451 - 517, 1983

${ }^{23}$ D.G. Crighton and F.G. Leppington, Radiation Properties of the Semi-Infinite Vortex Sheet: the Initial-Value Problem, Journal of Fluid Mechanics 64(2), 393-414, 1974 
${ }^{24}$ D.C. Pridmore-Brown, Sound Propagation in a Fluid Flowing through an attenuating Duct, Journal of Fluid Mechanics, 4, 393-406, 1958

${ }^{25}$ S.W. Rienstra, Impedance Models in Time Domain, including the Extended Helmholtz Resonator Model, 12th AIAA/CEAS Aeroacoustics Conference, 8-10 May 2006, Cambridge, MA, USA AIAA Paper 2006-2686.

${ }^{26}$ P. Huerre and P.A. Monkewitz, Absolute and convective instabilities in free shear layers, Journal of Fluid Mechanics, 159, 151-168, 1985.
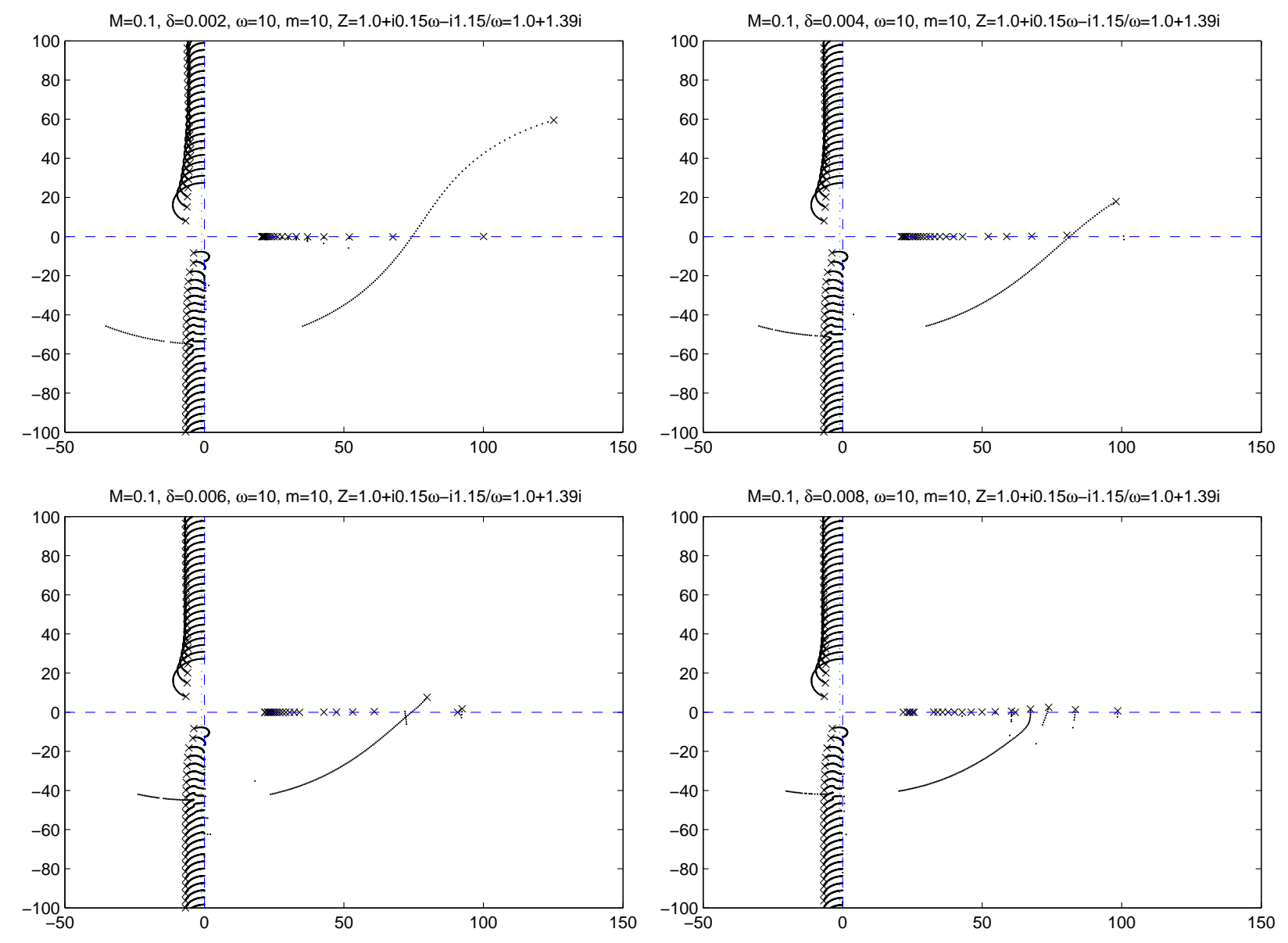

Figure 4. $M_{0}=0.1, \omega=10, m=10, Z=1+1.39 \mathrm{i}, \delta_{c} \simeq 0.008$. 

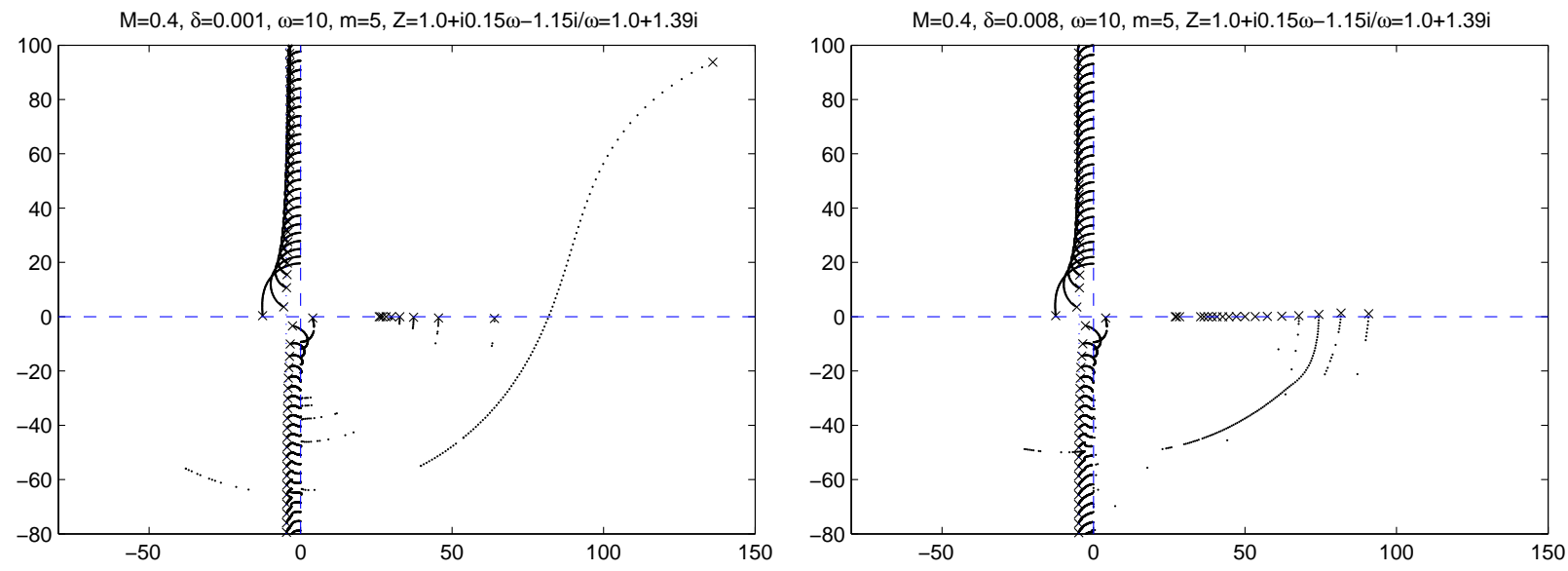

Figure 5. $M_{0}=0.4, \omega=10, m=5, Z=1+1.39 \mathrm{i}, \delta_{c} \simeq 0.008$
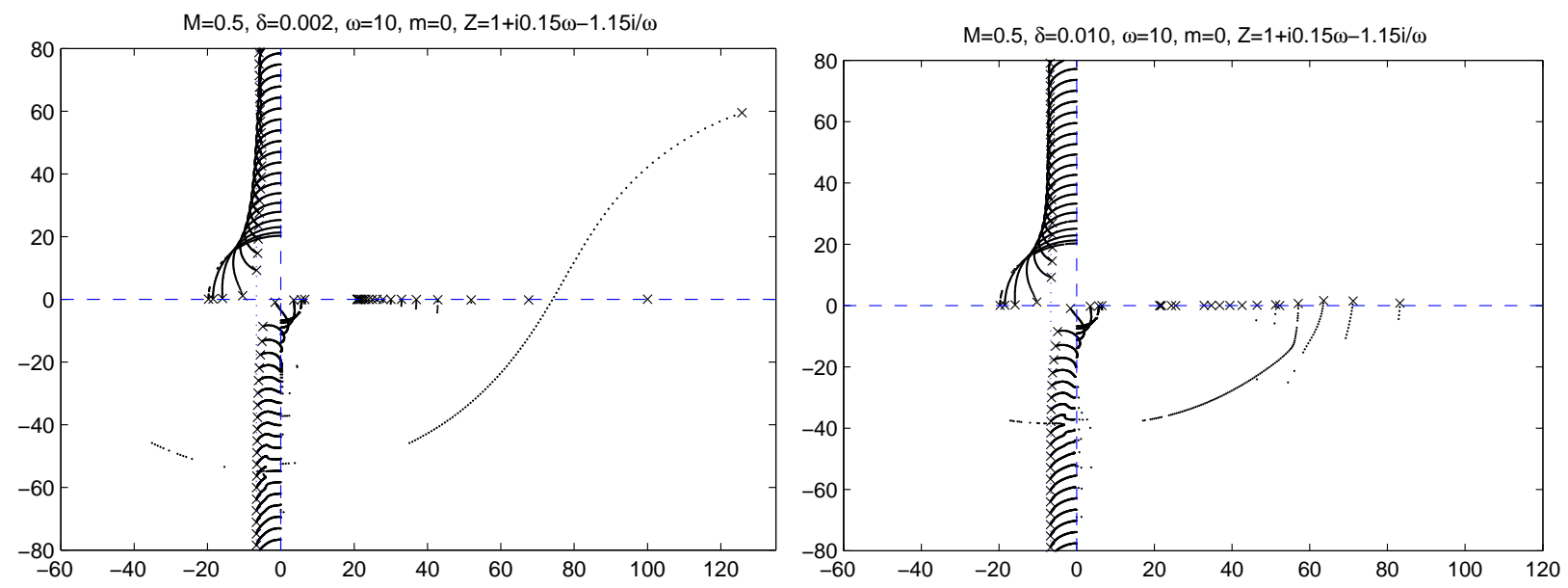

Figure 6. $M_{0}=0.5, \omega=10, m=0, Z=1+1.39 \mathrm{i}, \delta_{c} \simeq 0.010$
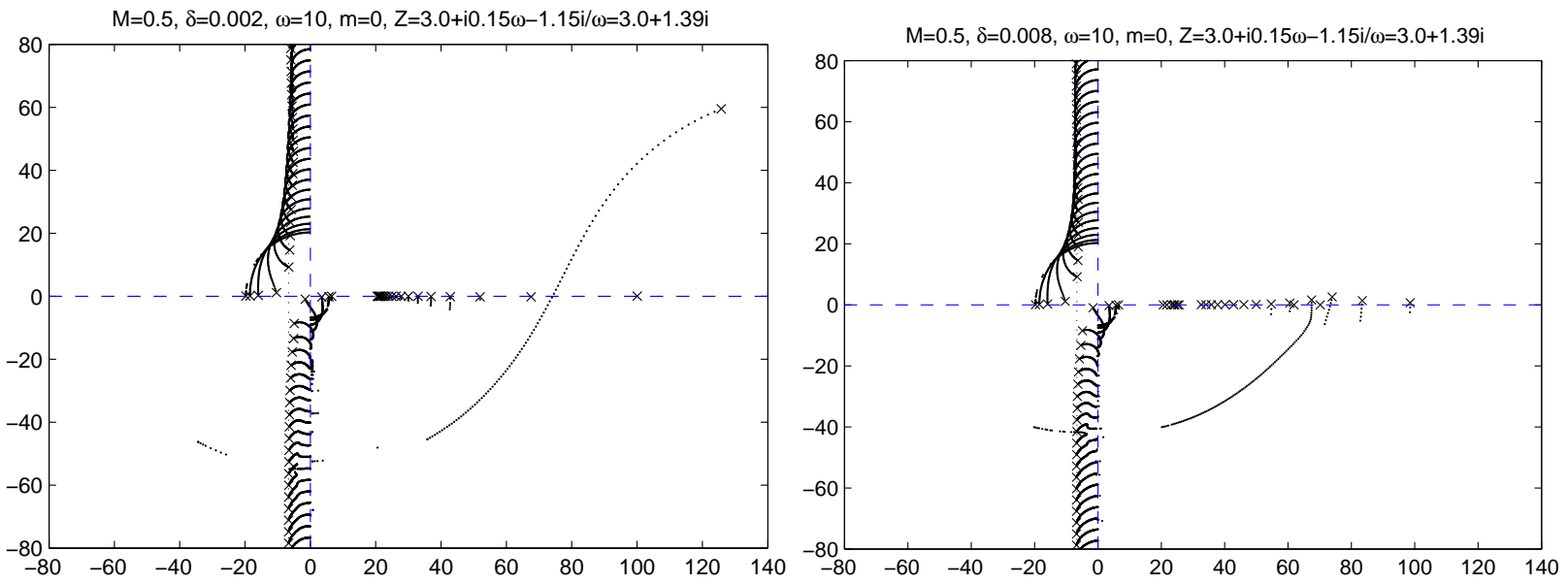

Figure 7. $M_{0}=0.5, \omega=10, m=0, Z=3+1.39 \mathrm{i}, \delta_{c} \simeq 0.008$ 

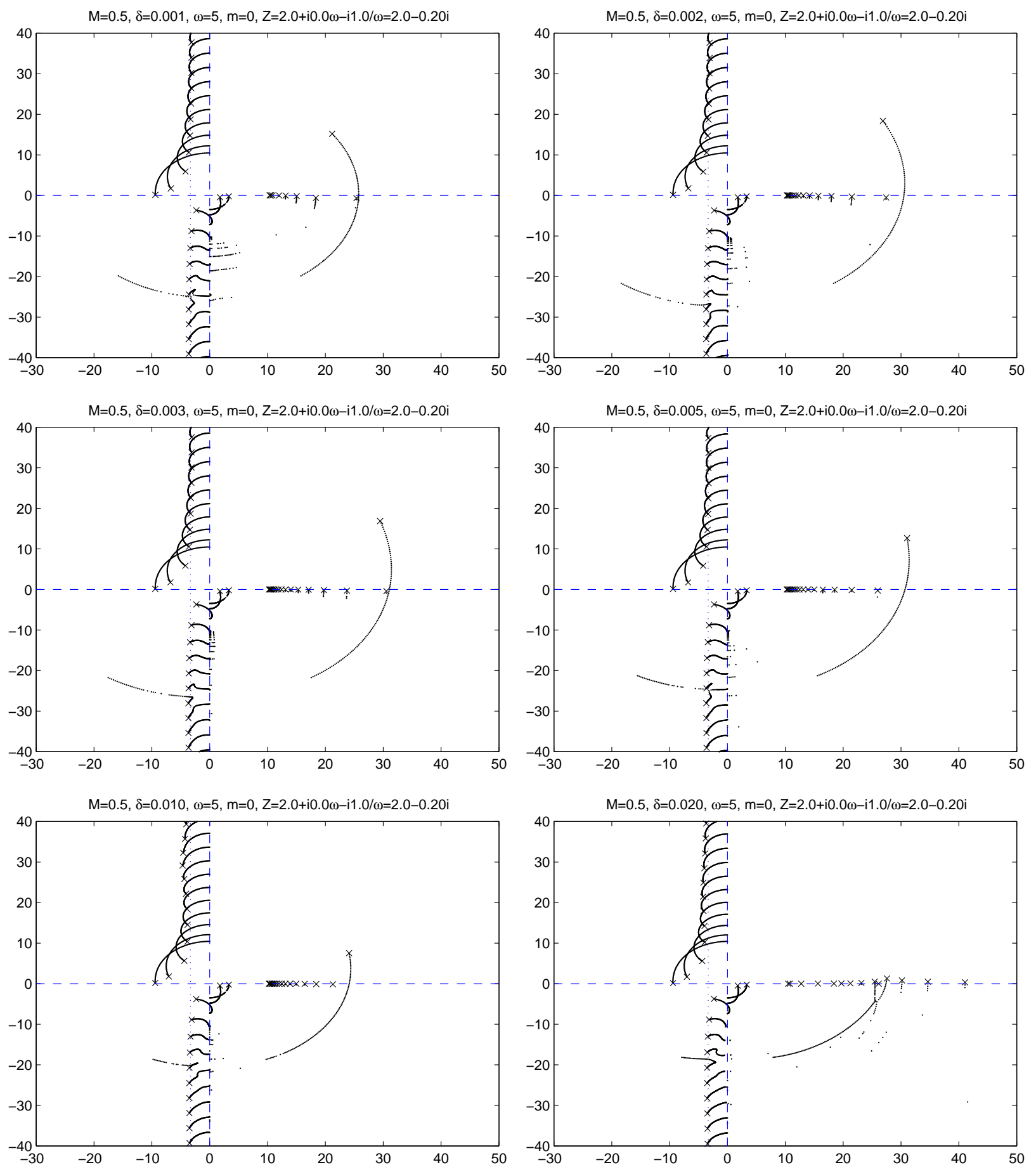

Figure 8. $M_{0}=0.5, \omega=5, m=0, Z=2-0.2 \mathrm{i}, \delta_{c} \simeq 0.020$. 

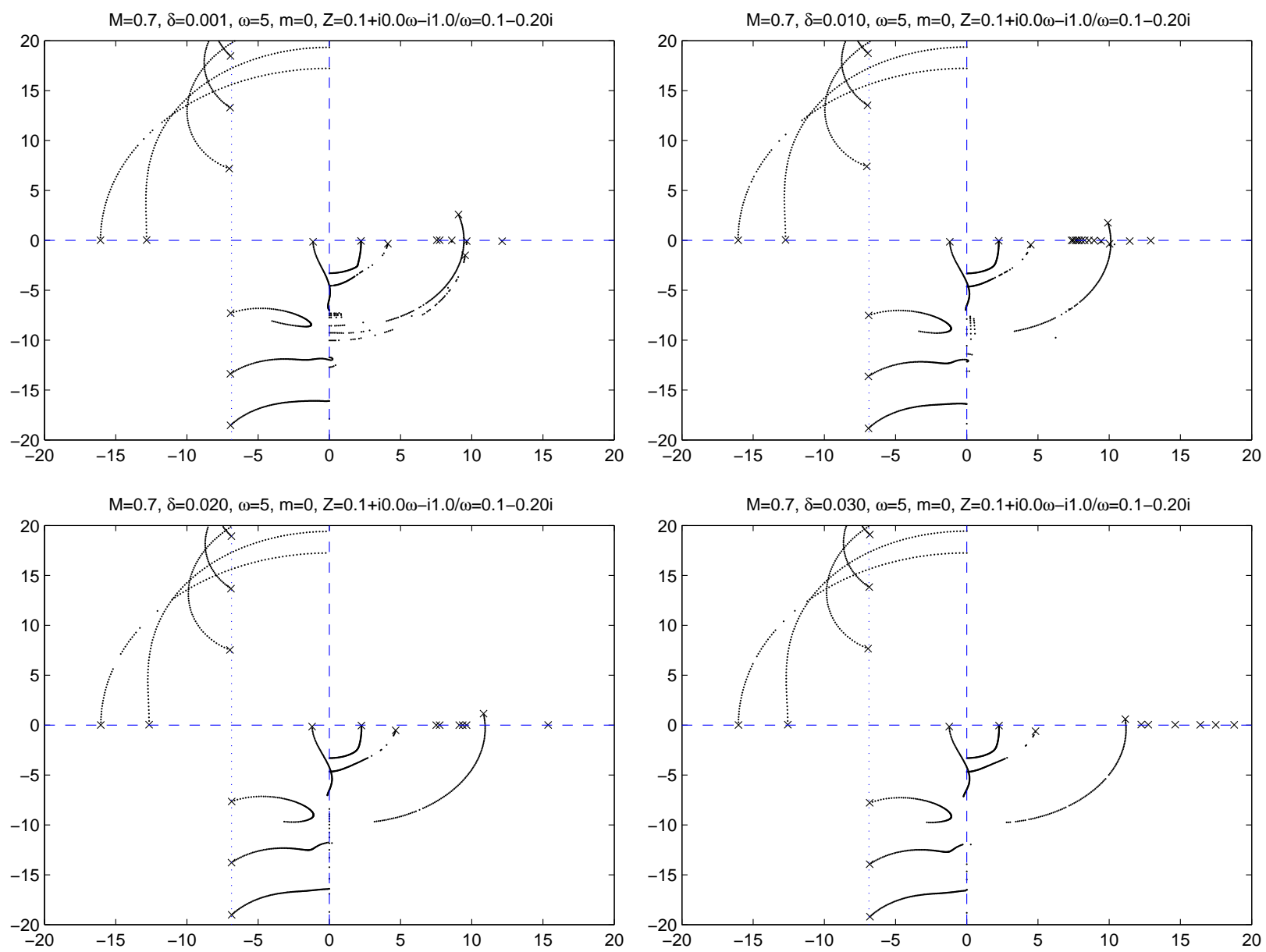

Figure 9. $M_{0}=0.7, \omega=5, m=0, Z=0.1-0.2 \mathrm{i}, \delta_{c} \simeq 0.030$. 

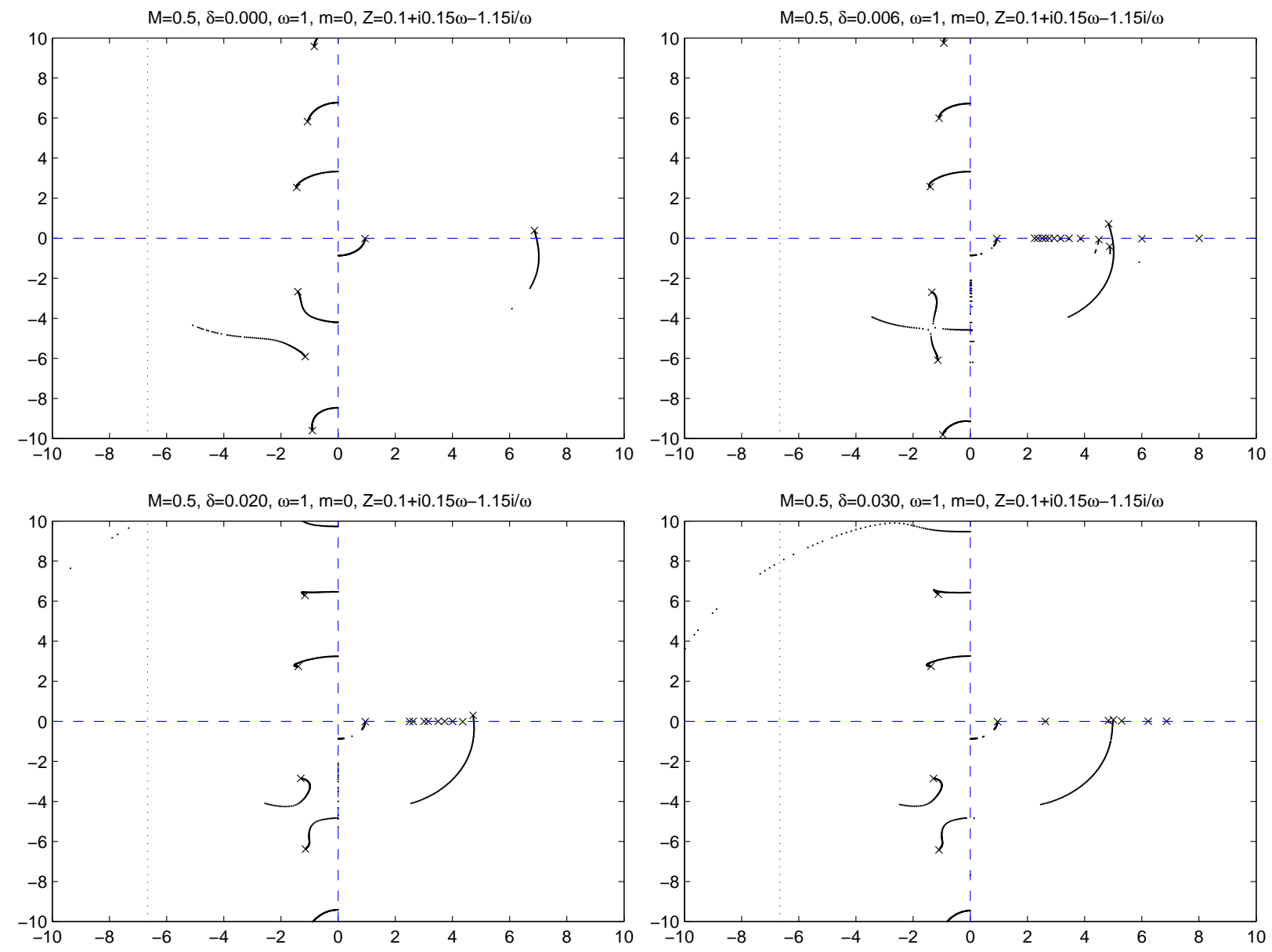

Figure 10. The unstable mode is effectively neutrally unstable for all $\delta\left(M_{0}=0.5, \omega=1, m=0, Z=0.1-\mathrm{i}\right)$
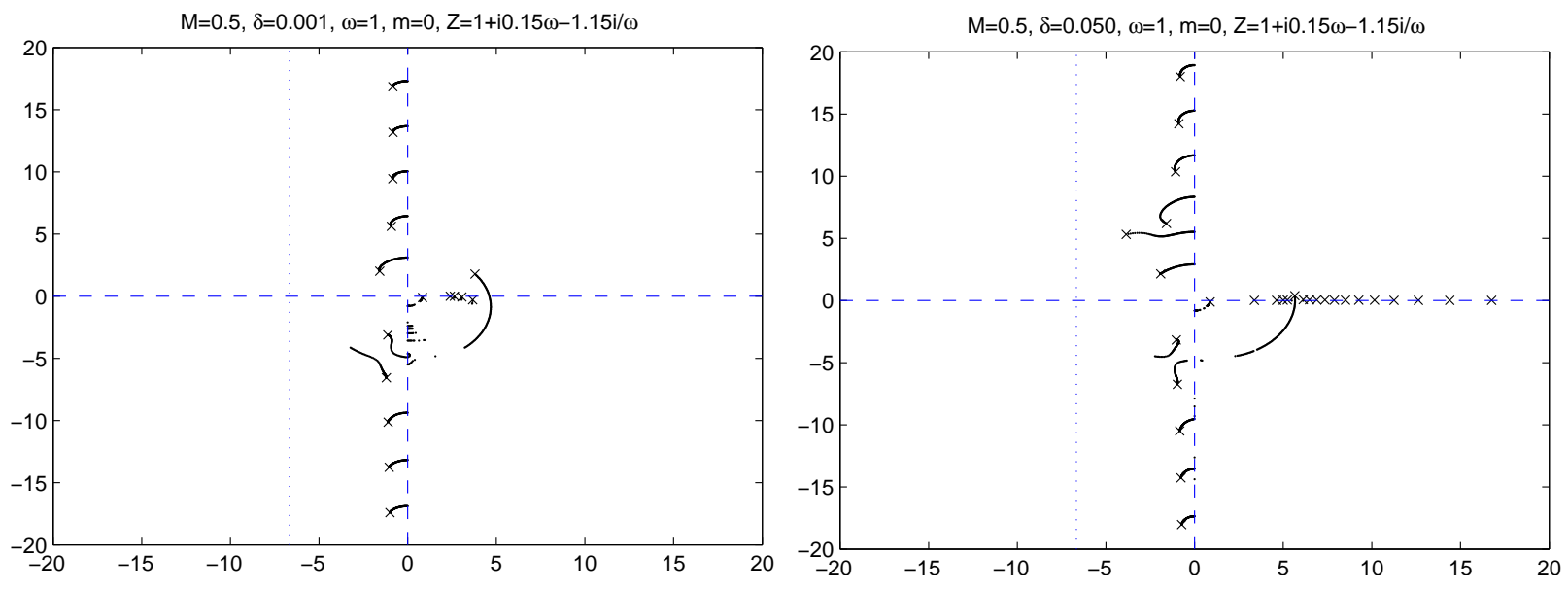

Figure 11. $M_{0}=0.5, \omega=1, m=0, Z=1-\mathrm{i}, \delta_{c} \simeq 0.05$ 
$\omega=10$
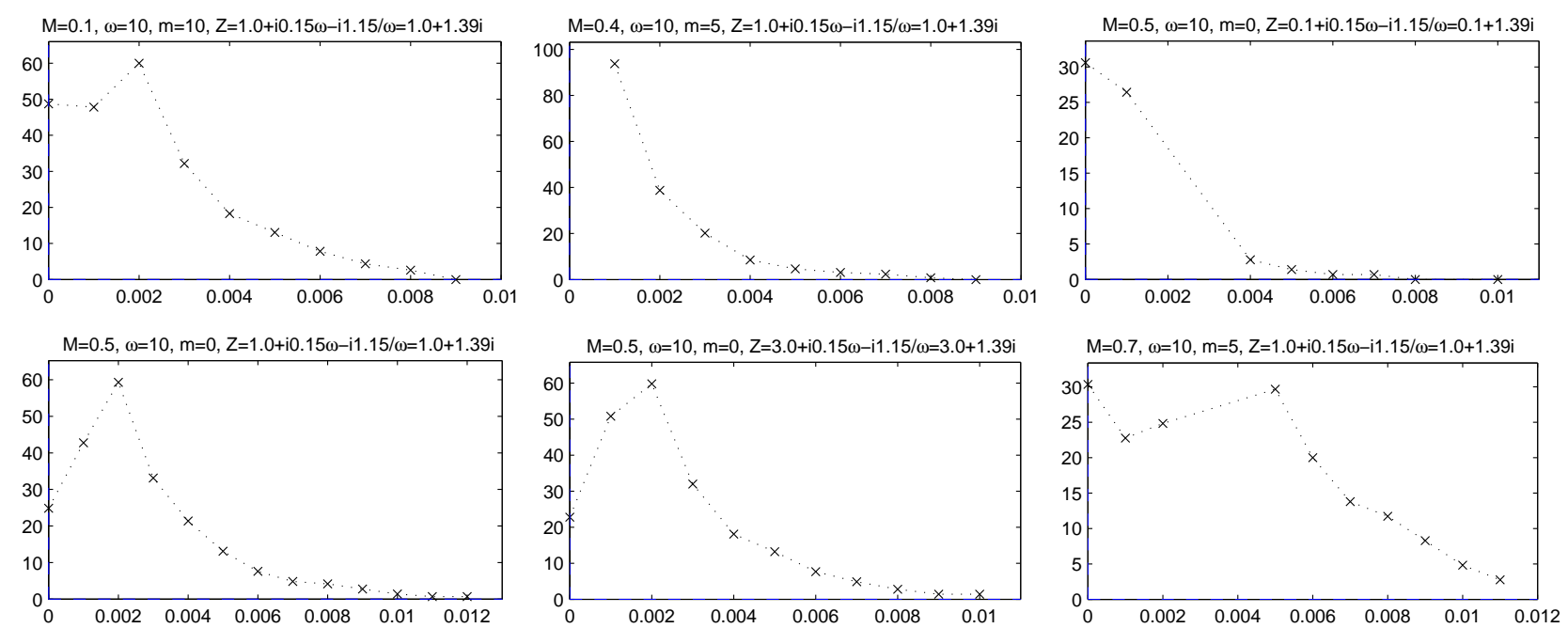

$\omega=5$
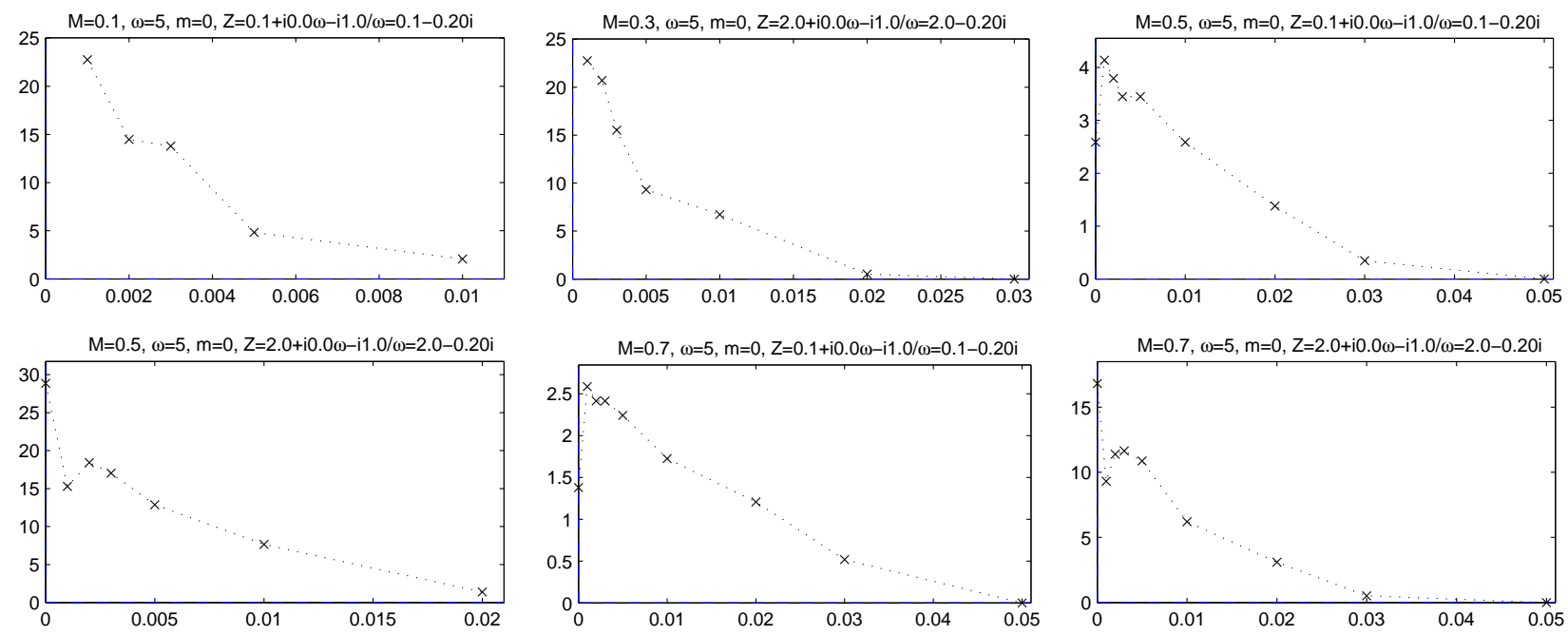

$$
\omega=1
$$
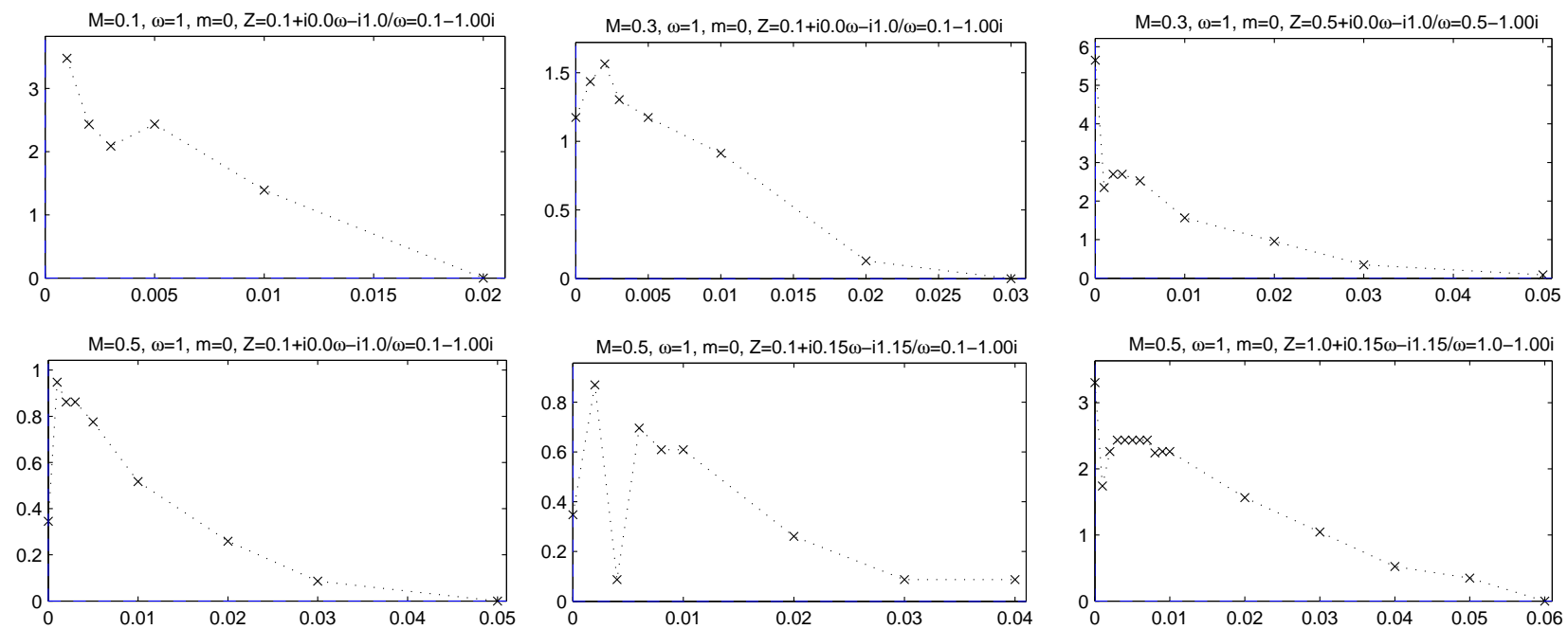

Figure 12. Imaginary parts $\operatorname{Im}(k)$ of the instability as a function of boundary layer thickness $\delta$

13 of 13

American Institute of Aeronautics and Astronautics Paper 2008-2932 